\title{
PAPER
}

\section{Apathy in Parkinson's disease}

\author{
G C Pluck, R G Brown
}

J Neurol Neurosurg Psychiatry 2002;73:636-642

\begin{abstract}
Objective: To assess apathy in patients with Parkinson's disease and its relation to disability, mood, personality, and cognition.

Methods: Levels of apathy in 45 patients with Parkinson's disease were compared with a group of 17 similarly disabled patients with osteoarthritis. Additional neuropsychiatric data were collected concerning levels of depression, anxiety, and hedonic tone. Personality was assessed with the tridimensional personality questionnaire. Cognitive testing included the mini-mental state examination, the Cambridge examination of cognition in the elderly, and specific tests of executive functioning. Results: Patients with Parkinson's disease had significantly higher levels of apathy than equally disabled osteoarthritic patients. Furthermore, within the Parkinson sample, levels of apathy appear to be unrelated to disease progression. The patients with Parkinson's disease with the highest levels of apathy where not more likely to be depressed or anxious than those with the lowest levels of apathy, though they did show reduced hedonic tone. No differences in personality traits were detected in comparisons between patients with Parkinson's disease and osteoarthritis, or between patients in the Parkinson group with high or low levels of apathy. As a group, the patients with Parkinson's disease tended not to differ significantly from the osteoarthritic group in terms of cognitive skills. However, within the Parkinson's disease sample, the high apathy patients performed significantly below the level of the low apathy patients. This was particularly evident on tests of executive functioning.

Conclusions: Apathy in Parkinson's disease is more likely to be a direct consequence of disease related physiological changes than a psychological reaction or adaptation to disability. Apathy in Parkinson's disease can be distinguished from other psychiatric symptoms and personality features that are associated with the disease, and it is closely associated with cognitive impairment. These findings point to a possible role of cognitive mechanisms in the expression of apathy.
\end{abstract}

See end of article for authors' affiliations

T he clinical significance of negative symptoms such as apathy is increasingly recognised in neurological and psychiatric disorders, particularly those associated with frontalsubcortical dysfunction. ${ }^{1}$ Apathy refers to a constellation of behavioural, emotional, and motivational features including a reduced interest and participation in normal purposeful behaviour, lack of initiative with problems in initiation or sustaining an activity to completion, lack of concern or indifference, and a flattening of affect. Parkinson's disease is a classic example of a subcortical disorder where apathy is observed. ${ }^{2-8}$ Evidence from other clinical groups suggests that the presence of apathy in patients poses difficulties for clinical management and care. Almost by definition, apathy has a direct impact on the overall level of handicap, as it reduces participation in age appropriate activities above and beyond that due to other aspects of the disease. It contributes significantly to carer burden ${ }^{10}$ and has negative implications for treatment and long term outcome. ${ }^{11-13}$ In Parkinson's disease, there is no evidence that the symptoms of apathy are improved by levodopa treatment, although there are early indications that the dopamine D3 receptor agonist, pramipexole, ${ }^{14}{ }^{15}$ and cholinesterase inhibitors may be of some value. ${ }^{16}{ }^{17}$

Current estimates of the prevalence of apathy in Parkinson's disease vary between $16.5 \%{ }^{6}$ and $42 \%,{ }^{2}$ depending upon the instrument used for assessment and on the samples examined. One drawback in existing reports is the lack of comparative data from elderly non-neurologically disabled samples. While it is generally assumed that apathy is a neuropsychiatric symptom directly related to underlying pathophysiology, ${ }^{6}$ it is also possible that some aspects reflect a direct response or even a psychosocial adaptation to the limitations imposed by long term physical disability. ${ }^{18}$

Although considered a distinctive symptom, apathy overlaps both conceptually and clinically with a range of other behavioural and psychological factors. These include mood (particularly depression), anhedonia, aspects of personality, and cognitive function. These will be discussed briefly in turn.

One of the most important issues for diagnosis and management is the relation between depression and apathy. Existing evidence suggests that the two are correlated in Parkinson's disease, ${ }^{5}$ but the symptoms can dissociate within individual patients. ${ }^{268}$ Whether instances of association are true comorbidity or overlap of individual symptoms at a superficial level is not yet clear. Apathy and depression more clearly dissociate in other disorders such as progressive supranuclear palsy, in which there is a high incidence of apathy but a low incidence of depression. ${ }^{19}$

Anxiety is also common in Parkinson's disease, ${ }^{20}$ but less clearly associated with apathy. ${ }^{2}$ Disinterest and lack of concern - a feature of apathy—seem to be incompatible with increased attention to events or "hypervigilance" seen in anxiety. ${ }^{21}$ However, using a factor analysis approach it has been found that in patients with Parkinson's disease apathy and anxiety scores on the neuropsychiatric inventory ${ }^{22}$ were correlated and clustered into one factor.

Anhedonia, the inability to experience pleasure, is a concept closely related to both apathy and depression..$^{23}$ In comparative

Abbreviations: $A D L$, activities of daily living; AES-C,

clinician/researcher rated apathy evaluation scale; AES-S, self report rated apathy evaluation scale; $\mathrm{BDI}$, Beck depression inventory;

CAMCOG, Cambridge examination of cognition in the elderly; COWAT, controlled word association test; HADS, hospital anxiety and depression scale; MMSE, mini-mental state examination; NS, novelty seeking; PD-HA, Parkinson's disease-high apathy; PD-LA, Parkinson's disease-low apathy; SE, Schwab and England scale; SHPS, Snaith-Hamilton pleasure scale; TPQ, tridimensional personality questionnaire; WCST, Wisconsin card sort test 
Table 1 Demographic characteristics and apathy evaluations of the Parkinson's disease and osteoarthritis groups

\begin{tabular}{|c|c|c|c|c|c|c|}
\hline Variable & $O A$ & PD & $\mathrm{p}$ Value & PD-LA & PD-HA & p Value \\
\hline Age & $67.3(9.7)$ & $66.4(8.6)$ & NS & $65.7(8.4)$ & $67.5(9.0)$ & NS \\
\hline Sex ratio $(M: F)$ & $5: 12$ & $22: 23$ & NS & $13: 15$ & $9: 8$ & NS \\
\hline Years of education & $11.2(2.5)$ & 13.00 (2.9) & $<0.05$ & $13.2(3.0)$ & $12.5(2.5)$ & NS \\
\hline Duration of PD & - & $10.3(6.0)$ & - & $9.9(5.3)$ & $10.9(7.4)$ & NS \\
\hline ADL & 43.7 (16.5) & $53.45(21.4)$ & $<0.1$ & $52.4(21.1)$ & $55.3(22.4)$ & NS \\
\hline SE & $80.6(15.1)$ & $73.1(15.9)$ & NS & 76.1 (15.5) & $68.2(15.9)$ & NS \\
\hline AES-S & $26.9(5.0)$ & $33.0(9.0)$ & $<0.05$ & $29.5(6.7)$ & $39.0(9.6)$ & $<0.05$ \\
\hline AES-C & $23.3(3.8)$ & 35.3 (11.4) & $<0.001$ & $28.0(4.6)$ & $47.8(7.9)$ & $<0.001$ \\
\hline
\end{tabular}

research, the dopamine system-particularly projections between the ventral tegmental area and the nucleus accumbens-has been identified as being closely associated with reward. ${ }^{24}$ This dopamine pathway is also impaired in Parkinson's disease ${ }^{25} 26$ and so it has been hypothesised that the processing of reward is hindered in patients with Parkinson's disease. ${ }^{27}$ This could manifest itself clinically as reduced hedonic tone and contribute to the phenomenology of apathy.

In contrast to such state changes in mood, trait factors have also been implicated in Parkinson's disease. A so called "parkinsonian personality" — characterised by inflexibility, moral rigidity, and introversion-has been described ${ }^{29}{ }^{30}$ and is considered to be directly related to the underlying disease pathophysiology. ${ }^{31}$ Personality theorists such as Cloninger have sought to integrate neurobiological models of neurotransmitter systems with specific aspects of personality such as novelty seeking and reward dependency. ${ }^{32}$ This raises the possibility that apathy and specific personality traits in Parkinson's disease may reflect the same neuropathological substrate. To date, this association has not been assessed.

The final factor to consider in relation to apathy is cognition, and particularly executive disorder, as this is thought to be impaired in many patients with Parkinson's disease. ${ }^{33}$ Individual studies have shown differentially impaired cognition and particularly executive dysfunction in apathetic patients with Alzheimer's disease, ${ }^{34}$ HIV infection, ${ }^{35}$ and Parkinson's disease. ${ }^{2}{ }^{3}$ Further study is necessary to confirm the generality of such a finding.

Based on such existing knowledge, our aim in this study was to address the following issues: first, whether apathy can be understood best as a reaction to disability or as a direct symptom of Parkinson's disease; second, the relation between apathy in Parkinson's disease and other psychiatric symptoms and aspects of personality; and third, the relation between apathy and cognitive impairment.

\section{METHODS}

In order to answer the questions posed, 62 patients with either Parkinson's disease $(n=45)$ or osteoarthritis $(n=17)$ were assessed on a range of neuropsychiatric, cognitive, and personality measures. All participants gave informed consent and were assessed in their own homes. Patients from both clinical groups were visited in the same time frame and were all assessed by the same investigator (GCP). Ethical approval was granted for the investigation of each patient group from the appropriate hospital ethics committees.

\section{Participants}

The Parkinson's disease patients were all current or past patients of the National Hospital for Neurology and Neurosurgery in London. They were recommended as probable cases of idiopathic Parkinson's disease by a consultant neurologist specialising in movement disorders. They were not selected for any known cognitive or psychiatric state. The sample included a wide range of disease progression, from Hoehn and Yahr ${ }^{36}$ stage 1 to 5 (mean 2.6). The osteoarthritis patients were all current or past patients of the department of rheumatology, Kings College Hospital, London. Appropriate patients where recommended by a consultant rheumatologist and were not selected on the basis of psychological factors. Characteristics of the two groups are shown in table 1.

\section{Assessment instruments}

The assessment of the two groups was identical with the exception that the Hoehn and Yahr disease progression staging was measured only in the Parkinson's disease sample. Disability was assessed by a self report activities of daily living (ADL) scale used in previous studies comparing Parkinson's disease and osteoarthritis patients, ${ }^{37}$ and on the Schwab and England scale (SE).$^{38}$ While this latter scale is generally used only in Parkinson's disease, it provides a useful global rating (0-100) of independence and performance on instrumental activities of daily living and is therefore of value in comparing the two samples.

Apathy was assessed using the clinician/researcher rated version of the apathy evaluation scale (AES-C), ${ }^{39}$ and the parallel self report version of the same instrument (AES-S).

Global cognitive function was assessed with the Cambridge examination of cognition in the elderly (CAMCOG $)^{40}$ and the mini-mental state examination (MMSE) ${ }^{41}$ The former test provides a more thorough assessment of cognition and has been shown to be useful in the identification of dementia in Parkinson's disease. ${ }^{42}$ To supplement these measures, three tests of executive function were included: the modified Wisconsin card sort test (WCST), ${ }^{43}$ the Stroop interference task ${ }^{44}$ and a test for verbal fluency (the controlled word association test, "COWAT"). ${ }^{45}$ Category fluency (animal names)-a part of the CAMCOG-was also analysed separately.

Mood was assessed using the hospital anxiety and depression scale (HADS) ${ }^{46}$ and the Beck depression inventory (BDI) ${ }^{47}$ Although the BDI has been criticised for use in Parkinson's disease samples, ${ }^{48}$ it is the most widely used self report depression scale in Parkinson's disease research and has the advantage of having been previously used to compare patients with Parkinson's disease and osteoarthritis. ${ }^{37}$ Hedonic tone was assessed with the Snaith-Hamilton pleasure scale (SHPS). ${ }^{50}$ This test was scored on all four points of a Likert scale, as well as the binary scoring system employed in the original paper. Personality was assessed using the tridimensional personality questionnaire (TPQ), ${ }^{32}$ which measures three personality dimensions-novelty seeking, harm avoidance, and reward dependence; an additional subscale of persistence was derived from the reward dependence scale.

Owing to fatigue and time constraints, some assessments were not completed by all patients. The lowest completion 


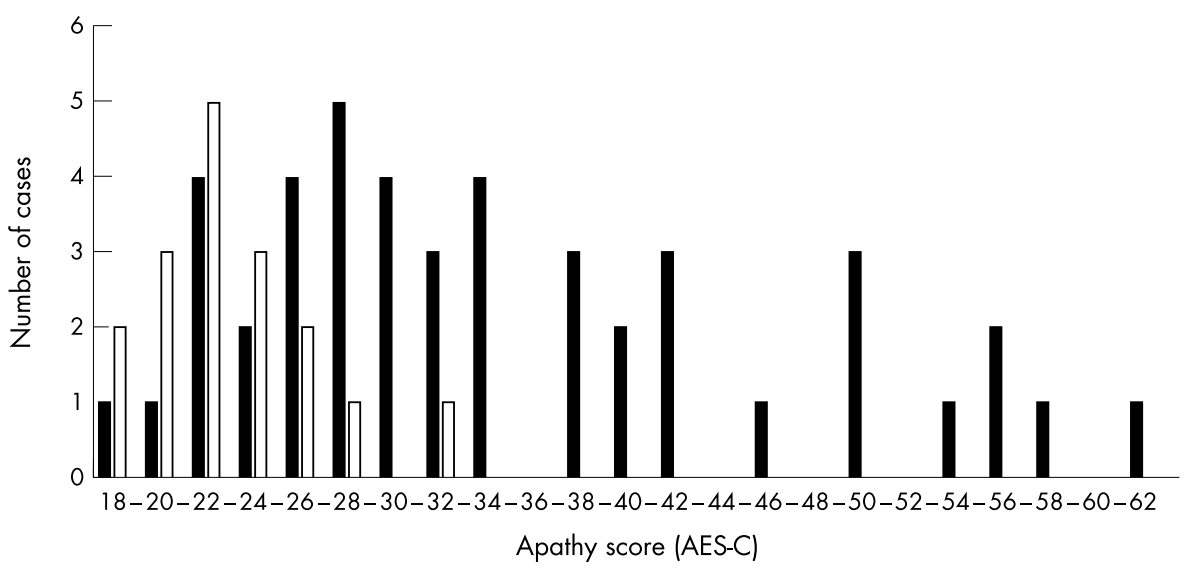

Figure 1 The distribution of apathy scores for patients with Parkinson's disease (black bars) and patients with osteoarthritis (grey bars).

rates were for the WCST and HADS, which are available for only $84 \%$ of participants.

\section{RESULTS}

Two independent sets of comparisons were undertaken. First, between the total Parkinson's disease sample and the osteoarthritis group. Second, within the Parkinson's disease group between those identified as having high and relatively low levels of apathy, according to the AES-C. The data and results related to these analyses are presented together in the tables. The data were analysed using analysis of variance (ANOVA; univariate, multivariate, and repeated measures as appropriate). Where indicated, demographic variables were used as constant covariates in the analyses.

\section{Group characteristics}

Characteristics of the Parkinson's disease and osteoarthritis groups are shown in table 1 . There were no significant differences between the mean ages of the groups. While not significant, there was a higher proportion of female patients in the osteoarthritis group. The Parkinson group had received more years of education, and so this variable was used as a covariate in subsequent comparisons related to cognitive function. The Parkinson group also tended to be more disabled and to have lower overall levels of independence, as measured by the ADL and SE scales. Despite the marginal levels of significance, it was decided to use the latter measure as a constant covariate in subsequent analyses of apathy, mood, and personality. Whether assessed by the researcher or by self report, the Parkinson group showed higher levels of apathy. In neither instance, however, was disability a significant covariate.

To check reliability, 20 patients with Parkinson's disease completed the AES-S at a second session three to six months after the initial assessment. The correlation between the two administrations was high $(r=0.85, \mathrm{p}<0.001)$. The AES-S and AES-C were found to be highly correlated ( $r=0.74$, $\mathrm{p}<0.001$ ). As it has previously been shown that the AES-C has greater predictive validity, ${ }^{39}$ further analysis was limited to this measure. The internal consistency of the AES-C was also assessed within the Parkinson group and found to be high $(\alpha=0.92)$.

The distribution of apathy scores for the Parkinson's disease and osteoarthritis patients is shown in fig 1. A cut off point of 38 has been used previously to identify clinically significant apathy. ${ }^{51}$ This was used to divide the Parkinson's disease patients into two groups-a "high apathy" group (PD-HA) containing 17 patients $(37.8 \%)$ and a "low apathy" group (PD-LA) contained 28 patients $(62.2 \%)$. Classification based on the self report data produced the same results with $36 \%$ rating themselves as apathetic. None of the osteoarthritis patients was identified as apathetic using the AES-C, compared with one patient using the AES-S. These findings are consistent with existing normative data. ${ }^{39}$

Comparisons of the PD-HA and PD-LA samples showed no significant differences in age, sex ratio, years of education, or self reported ADL. The PD-HA group tended to have a slightly longer duration of disease and appeared to more dependent, as measured by the SE scale, but these differences were not significant.

\section{Cognitive performance}

Mean scores for the cognitive assessments are shown in table 2. Controlling for level of education the Parkinson's disease group as a whole showed little in the way of global cognitive impairment compared with the osteoarthritis group. The patients with Parkinson's disease appeared to be impaired on the category fluency task, but this needs to be interpreted with caution in the light of the number of individual analyses undertaken. On the Stroop test, a repeated measures analysis of variance showed that the Parkinson group was slower overall across conditions $(p<0.05)$, with a significant group $\times$ condition interaction $(p<0.05)$. Post hoc comparisons revealed significant impairment for reading and colour naming but not for the interference condition.

Comparison of the PD-HA and PD-LA subgroups showed more cognitive differences. While the MMSE was not sensitive, a significant group difference was found on the CAMCOG. Of the PD-HA group, 3/16 patients scored below the recommended cut off score of 80 , while none of the PD-LA group scored below this level. CAMCOG data were missing on one PD-HA patient, but his performance on other cognitive tests suggested the presence of clinically significant cognitive impairment. In all, therefore, 4/17 (23.5\%) of the high apathy subgroup could be considered demented on the basis of cognitive testing.

The CAMCOG subscales were assessed together using a single multivariate ANOVA. Significant univariate differences were found for language and memory. Relative impairment was displayed on both fluency tasks, but only with letter fluency (COWAT) was this difference significant. Performance on the WCST was more impaired in the PD-HA group in terms of categories sorted and the number of errors. In terms of perseverative errors the impairment was less marked, with no difference in the percentage of such errors. Overall, the PD-HA group was impaired on the Stoop test $(p<0.01)$, with a significant group $\times$ condition interaction $(p<0.05)$. Post hoc tests showed significant impairments under all conditions, but particularly interference.

Mood, hedonia, and personality

Mean scores derived from the assessments of depression, anxiety, hedonic tone, and personality are shown in table 3. 
Table 2 Scores for Parkinson's disease and osteoarthritis groups on tests of cognitive function

\begin{tabular}{|c|c|c|c|c|c|c|}
\hline & OA & PD & $\mathrm{p}$ Value & PD-LA & PD-HA & $\mathrm{p}$ Value \\
\hline MMSE & $27.6(2.9)$ & $27.8(2.4)$ & NS & 28.1 (1.9) & $27.3(3.1)$ & NS \\
\hline CAMCOG - total & 92.7 (9.9) & $93.0(6.4)$ & NS & 94.7 (4.2) & $90.1(8.3)$ & $<0.05$ \\
\hline Orientation & $9.6(0.6)$ & $9.7(0.7)$ & NS & $9.7(0.7)$ & $9.6(0.7)$ & NS \\
\hline Language & $27.0(2.3)$ & $7.0(2.4)$ & NS & $27.7(0.9)$ & $25.9(3.6)$ & $<0.05$ \\
\hline Attention & $5.8(1.5)$ & $5.9(1.6)$ & NS & $5.7(1.6)$ & $6.1(1.6)$ & NS \\
\hline Memory & $21.8(2.8)$ & $21.6(2.7)$ & NS & $22.4(2.1)$ & $20.3(3.0)$ & $<0.05$ \\
\hline Praxis & 10.9 (1.5) & $11.2(1.5)$ & NS & 11.5 (1.1) & $10.6(1.9)$ & $<0.10$ \\
\hline Calculation & $1.9(0.3)$ & $2.0(0.2)$ & NS & $2.0(0.0)$ & $1.9(0.3)$ & $<0.10$ \\
\hline Abstract reasoning & $6.4(1.4)$ & $7.0(1.0)$ & NS & $7.1(0.8)$ & $6.9(1.1)$ & NS \\
\hline Perception & $9.0(1.5)$ & $8.5(1.3)$ & NS & $8.7(1.3)$ & $8.3(1.4)$ & NS \\
\hline COWAT* & 10.8 (4.89) & 12.0 (3.9) & NS & $13.1(3.7)$ & $10.2(3.7)$ & $<0.05$ \\
\hline Category fluency & $19.4(4.26)$ & 16.9 (4.9) & $<0.05$ & $18.1(4.6)$ & $15.2(4.9)$ & $<0.10$ \\
\hline WCST & & & & $(n=26)$ & $(n=11)$ & \\
\hline Categories & $5.0(1.7)$ & $4.5(2.1)$ & NS & $5.0(1.4)$ & $3.4(2.7)$ & $<0.05$ \\
\hline Total errors & $7.4(7.7)$ & $11.7(10.4)$ & NS & $10.1(8.1)$ & $15.5(14.3)$ & $<0.05$ \\
\hline Perseverative errors & $3.3(3.1)$ & $4.6(5.5)$ & NS & $3.9(4.3)$ & $6.4(7.7)$ & NS \\
\hline$\%$ Perseverative errors & $41.6(33.6)$ & $31.4(22.2)$ & NS & $31.5(22.9)$ & $31.2(21.6)$ & NS \\
\hline \multicolumn{7}{|l|}{ Stroop test $†$} \\
\hline Reading & $93.4(19.6)$ & $81.6(16.2)$ & $<0.05$ & $87.5(14.5)$ & $71.8(14.3)$ & $<0.01$ \\
\hline Colour naming & $67.8(17.2)$ & $57.6(11.8)$ & $<0.05$ & $59.7(12.4)$ & $53.9(9.9)$ & $<0.05$ \\
\hline Interference & $31.9(8.8)$ & $29.4(9.9)$ & NS & $32.9(8.3)$ & $23.5(9.9)$ & $<0.001$ \\
\hline \multicolumn{7}{|c|}{$\begin{array}{l}\text { Values are mean (SD). } \\
\text { *Mean number of items for letters F, A, and S. } \\
\text { †Number of items in } 45 \text { seconds. } \\
\text { NS }=p>0.10 \text {. } \\
\text { CAMCOG, Cambridge examination of cognition in the elderly; COWAT, controlled word association test; } \\
\text { MMSE, mini-mental state examination; OA, osteoarthritis group; PD, Parkinson's disease group; PD-HA, } \\
\text { Parkinson's disease-high apathy; PD-LA, Parkinson's disease-low apathy; WCST, Wisconsin card sort test. }\end{array}$} \\
\hline
\end{tabular}

Table 3 Scores for the Parkinson's disease and osteoarthrisits groups on assessments of mood (BDI and HADS), hedonic tone (SHPS), and personality (TPQ)

\begin{tabular}{|c|c|c|c|c|c|c|}
\hline & OA & PD & $\mathrm{p}$ Value & PD-LA & PD-HA & $\mathrm{p}$ Value \\
\hline BDI & $8.5(4.6)$ & $13.7(7.1)$ & $<0.05$ & $13.3(5.9)$ & $14.4(8.9)$ & NS \\
\hline \multicolumn{7}{|l|}{ HADS } \\
\hline Depression & $3.9(3.2)$ & $5.4(3.4)$ & NS & $4.8(2.7)$ & $6.5(4.2)$ & NS \\
\hline Anxiety & $6.7(5.1)$ & $8.6(4.5)$ & NS & $8.4(4.2)$ & $8.8(5.1)$ & NS \\
\hline SHPS & $21.7(4.0)$ & $23.2(4.8)$ & NS & $22.1(5.0)$ & $25.5(3.4)$ & $<0.05$ \\
\hline \multicolumn{7}{|l|}{$\mathrm{TPQ}$} \\
\hline Novelty seeking & $12.1(4.2)$ & $12.7(5.3)$ & NS & $12.6(5.2)$ & $12.9(5.8)$ & NS \\
\hline Harm avoidance & $16.5(7.1)$ & $19.2(7.1)$ & NS & $18.5(6.6)$ & $20.5(7.9)$ & NS \\
\hline Reward dependency & $17.8(3.7)$ & $17.2(4.7)$ & NS & $17.8(5.3)$ & $16.3(3.6)$ & NS \\
\hline Persistence & $4.5(1.9)$ & $4.2(2.2)$ & NS & $4.7(2.2)$ & $3.2(1.9)$ & $<0.10$ \\
\hline
\end{tabular}

Controlling for disability as measured by the SE scale, the total Parkinson's disease group was more depressed than the osteoarthritis group as assessed by the BDI but not by the HADS. Taking a cut off score of BDI $\geqslant 15,43 \%$ of the Parkinson's disease group had possible depression compared with $8 \%$ of the osteoarthritis group. However, using the recommended cut off of $\geqslant 11$ on the HADS, only two of the Parkinson group were identified as depressed (both apathetic) compared with one in the osteoarthritis group. No significant group differences were found for mean anxiety, although a large number in each group scored above the cut off score (Parkinson's disease $32 \%$, osteoarthritis $21 \%$ ). The Parkinson's disease and osteoarthritis groups did not differ in mean hedonic tone scores on the SHPS when measured on a Likert scale. Measur- ing on a binary scale and with a cut off of $\geqslant 3$, none of the osteoarthritis group was impaired, compared with three $(6.7 \%)$ from the Parkinson group. However, all but one scored just above the cut off, and all showed significant comorbid depressive symptomatology. The two groups did not differ on any of the personality scales assessed by the TPQ.

Comparing the two Parkinson's disease subgroups (low apathy with high apathy), no significant differences were found for the measures concerned with depression or anxiety. Of the PD-HA group, 44\% showed possible depression on the BDI, compared with $43 \%$ of the PD-LA group. Significant anxiety was present in $36 \%$ of the PD-HA group compared with $29 \%$ of the PD-LA group. Scores on the Lickert marked SHPS where significantly higher in the PD-HA group 
compared with the PD-LA group, indicating reduced hedonic tone in the apathetic patients. Of the three "anhedonic" patients, two were in the PD-HA group and one in the PD-LA group. As the scores on the SHPS scale where not normally distributed, non-parametric analyses were performed using Mann-Whitney U tests. The same overall results were obtained.

\section{Correlational and regression analyses}

Individual researcher rated apathy scores (AES-C) were analysed with the primary demographic, clinical, cognitive, mood, and personality measures in the Parkinson group using correlation statistics. Because of the number of pairwise comparisons, a conservative criterion was used for significance ( $\mathrm{p}<0.005, r>0.42$, two tailed test). Increasing apathy was associated with overall cognitive impairment as measured by the CAMCOG $(r=-0.50)$, and specifically with the subscales for language $(r=-0.46)$ and memory $(r=-0.49)$. An association was found between the degree of apathy and measures of executive function including letter fluency $(r=-0.42)$, Stroop reading $(r=-0.45)$ and interference $(r=-0.51)$, WCST categories $(r=-0.48)$ and perseverative errors $(r=0.43)$. A separate analysis of the associations with depression scores showed no significant relations for any of the above measures.

A series of exploratory regression analyses revealed that none of the clinical or demographic variables-alone or in combination (age, sex, education, duration of illness, Hoehn and Yahr stage or Schwab and England score)—predicted researcher rated apathy $(r=0.39, \mathrm{p}=0.23)$. Of the cognitive measures, the best set of predictors was the interference trial of the Stroop test and the COWAT verbal fluency $(r=0.55$, $\mathrm{p}=0.003)$.

\section{DISCUSSION}

Our study compared levels of apathy in a Parkinson's disease group with a non-neurological physical disease control group. The choice of osteoarthritis as an appropriate control group was based on the age range of the patient population and the facts that the condition is chronic, progressive, and causes significant levels of disablement. Although there was a trend for the Parkinson's disease patients to be more disabled than the osteoarthritis group, the differences were not significant, and the majority of patients showed mild to moderate levels of disability. In the introduction we raised the possibility that the symptoms of apathy may, in some way, be a consequence of chronic disabling disease and its impact on mobility and opportunity for participation in normal activities. Thus Singer ${ }^{18}$ used the term "premature social aging" to describe her findings that patients with Parkinson's disease, compared with otherwise healthy elderly individuals, have little in the way of interests or social activities, spending more time in solitary activities such as watching television or just sitting doing nothing. If such behavioural change is a primary consequence of physical disability and is a contributory factor to the clinical phenomenon of apathy, then similar changes might be predicted for patients with osteoarthritis. In fact, the results showed significant levels of apathy only in the Parkinson's disease sample. The osteoarthritis sample, despite their disability, showed no evidence of apathy. The mean (SD) score of the sample on the AES-C of 23.3 (3.8) was slightly less than data reported previously for a sample of healthy elderly subjects (26.0 (6.2)). ${ }^{39}$ Such results support the conclusion that apathy is a true feature of the Parkinson's disease process and not a psychological response to physical impairment and associated disability.

The AES was developed as a general measure of apathy in neurological patients. Because some of the items may overlap with the motor symptoms of Parkinson's disease (as in the assessment of depression) it is important to consider the validity of the AES as a measure of apathy. Several facts support its use. First, in the present study, the scale was found to have high internal consistency, suggesting that it was measuring a single construct. Second, the total apathy score was only weakly related or unrelated to disease duration, stage, or disability, factors which indicate the degree of motor impairment. Previous research has similarly failed to find any difference between apathy scores in patients with tremor dominant and akinetic-rigid dominant Parkinson's disease. ${ }^{52}$ Third, the strong association between apathy and cognitive function is in contrast to the majority of studies which find little relation between the severity of cognitive and motor symptoms. Finally, as an additional check on the data, a secondary analysis was carried out (not reported) whereby any AES item with potential confounding with general parkinsonian symptoms was removed. Using a cut off from the osteoarthritis group, 44 of 45 patients with Parkinson's disease obtained the same classification of high or low apathy as when using the complete scale. Such data support the use of the AES as a valid measure of apathy in Parkinson's disease.

No attempt was made to assess the relation between apathy and on/off phenomena within the Parkinson's disease sample. Such rapid motor fluctuations are common in advanced cases of the disease and can influence affective symptoms. ${ }^{53}$ However, in the present study apathy was assessed in relation to the individual's general levels of interests, motivation, emotional responsiveness, and so on, rather than their present state. Acute variations in apathy or its individual symptoms during on/off periods would be an interesting question to address, although more different assessment instruments may be required.

Returning to the present data, the distribution of apathy scores in the present study suggested that $37.8 \%$ of the Parkinson's disease patients were apathetic, a figure that is within the $16.5 \%$ and $42 \%$ range reported previously using self report or informant based measures. ${ }^{268}$ Although high, this estimate of $37.8 \%$ may be a significant underestimate of the population figure. Only $7.1 \%$ of the sample were identified as demented based on the CAMCOG score, compared to a figure of $47 \%$ in a community based sample reported previously. ${ }^{42}$ This latter sample contained a large number of elderly and institutionalised patients, typically ignored by the majority of research. Given the strong association between cognitive dysfunction and apathy, we would predict that the true figures for apathy within the Parkinson's disease population may be even higher than that indicated in the present study.

The Parkinson's disease sample as a whole showed higher levels of depression than the osteoarthritis group when measured with the BDI, although not with the HADS. More Parkinson's disease patients were identified as being above the cut off score for depression using the BDI, the later perhaps reflecting the differential sensitivity of the instruments for detecting depression in Parkinson's disease. Even using the BDI, however, there was no difference between the level of depression in the high and low apathy Parkinson's disease groups, and no association between the depression and apathy scores. While comorbid depression and apathy have an additive effect on symptoms such as cognitive dysfunction, it seems unlikely that depression is a major confounding factor in interpreting the present results.

As with depression, comparable levels of anxiety were found in the apathetic and non-apathetic patients. The previous research on this issue has been inconclusive: although in one study anxiety was found to be associated with apathy in Parkinson's disease patients, ${ }^{6}$ in another no relation was detected. ${ }^{2}$ Our present study employed for the first time an independent measure of hedonic tone. Using the standard criterion for the scale used,,$^{50} 6.7 \%$ of the Parkinson's disease sample were classified as "anhedonic," although there was no overall difference between the osteoarthritic and Parkinson's disease groups in hedonic tone. However, mean levels were 
significantly reduced in the high apathy group compared with the low apathy group, supporting the idea that loss of enjoyment or pleasure is a feature of the condition.

In order to investigate the possible role of personality in the expression of apathy, an inventory based on a neurochemical model of personality was used. ${ }^{32}$ The trait of particular relevance is novelty seeking (NS), as it has been argued that this is based on dopaminergic tone within the CNS. ${ }^{54}$ In a previous study, NS scores were found to be lower in Parkinson's disease patients ${ }^{55}$ than in medical controls. The current study failed to replicate this. More importantly, for the questions being addressed there was no significant difference in NS scores between Parkinson's disease patients with high and low apathy scores, nor for the other three personality traits assessed-harm avoidance, reward dependence, and persistence. However, for the latter, the result was approaching significance. The current findings, therefore, indicate that the hypothesised personality style in people with Parkinson's disease may not be an inevitable feature of the disease, nor related to the presence or absence of apathy.

The most salient results in this study concerned the relation between apathy and cognition. It has previously been shown that executive function tends to be differentially impaired in Parkinson's disease patients displaying apathy. ${ }^{23}$ Our study replicated those findings, with the high apathy patients performing below the level of the low apathy subgroup on various measures of executive function. On one measure of verbal fluency, a classical test of "willed action", 56 Parkinson's disease patients with high levels of apathy were highly significantly impaired, and there was a general slowness in output in the Stroop test. Basic visual processing has been shown to be impaired in subtle ways in Parkinson's disease, probably because of dopamine deficiency in the retina and primary visual cortex. ${ }^{57}$ This may therefore predispose to lower overall scores in the Parkinson's disease groups. However, this would not explain the differential impairment of the high apathy group on the interference task, which completed only $71 \%$ as many items as the low apathy group, compared with $90 \%$ in the colour naming condition. Deficits were also found on executive tasks that were less dependent on speed of visual processing, particularly the WCST, where the high apathy patients showed a marked reduction in the number of categories achieved. This was associated with an increase in the number of errors, although not an increased percentage of perseverative errors.

More general cognitive impairment in the apathetic patients was revealed by the CAMCOG. Memory function was affected, although some of this deficit might be related to executive processes, including working memory or encoding strategy, ${ }^{58}$ and performance on a range of memory tests correlate with executive dysfunction in Parkinson's disease. ${ }^{59}$ Similarly, scores on the language subscale of the CAMCOG were also reduced in the high apathy Parkinson's disease patients, although this scale includes a test of verbal fluency. More research will be needed to elucidate further any relation between apathy and non-executive aspects of cognition.

The cognitive, and particularly executive, dysfunction often reported in Parkinson's disease thus seems to be most evident in those patients who show the clinical features of apathy. While possible dementia was found in only a small proportion of the patients included in the present studies, it is significant that all of them were defined as apathetic - a result consistent with the classical description of fronto-subcortical dementia, ${ }^{60}$ where apathy is one of the defining neurobehavioural features. This raises the question of whether patients with marked apathy and associated cognitive dysfunction represent a distinct subgroup of the Parkinson's disease population or simply an extreme of a range of dysfunction. The extent to which the presence of apathy marks a subtype of Parkinson's disease patient may have wider implications for the prognosis and treatment of people with Parkinson's disease.

\section{ACKNOWLEDGEMENTS}

We are grateful to Professors Quinn and Scott for their expert advice on patient selection and assessment. This research was funded by a studentship to GCP granted by the Medical Research Council of England and Wales.

\section{Authors' affiliations}

G C Pluck, Division of Psychological Medicine, Section of Old Age Psychiatry, Institute of Psychiatry, King's College London, London SE5, UK R G Brown, Department of Psychology, Institute of Psychiatry

Competing interests: none declared

\section{REFERENCES}

1 Brown RG, Pluck G. Negative symptoms: the "pathology" of motivation and goal-directed behaviour. Trends Neurosci 2000;23:412-17.

2 Starkstein SE, Mayberg SE, Preziosi TJ, et al. Reliability, validity and clinical correlates of apathy in Parkinson's disease. J Neuropsychiatry 1992:4:134-9

3 Isella V, Melzi P, Grimaldi M, et al. Clinical, neuropsychological and morphometric correlates of apathy in Parkinson's disease. Move Disord 2002; 17:366-71

4 Ringman JM, Diaz-Olavarrieta C, Rodriguez $Y$, et al. The prevalence and correlates of neuropsychiatric symptoms in a population with Parkinson's disease in Mexico. Neuropsychiatry Neuropsychol Behav Neurol 2002;15:99-105.

5 Levy ML, Cummings JL, Fairbanks LA, et al. Apathy is not depression. J Neuropsychiatry Clin Neurosci 1998;10:314-19.

6 Aarsland D, Larsen JP, Goek Lim N, et al. Range of neuropsychiatric disturbances in patients with Parkinson's disease. J Neurol Neurosurg Psychiatry 1999;67:492-6.

7 Aarsland D, Cummings JL, Larsen JP. Neuropsychiatric differences between Parkinson's disease with dementia and Alzheimer's disease. Int J Geriatr Psychiatry 2001;16:184-91.

8 Aarsland D, Litvan I, Larsen JP. Neuropsychiatric symptoms of patients with progressive supranuclear palsy and Parkinson's disease. J Neuropsychiatry Clin Neurosci 2001;13:42-9.

9 Marsh NV, Kersel DA, Havill JH, et al. Caregiver burden at 1 year following severe traumatic brain injury. Brain Injury 1998;1 2:1045-59.

10 Thomas P, Clement JP, Hazif-Thomas C, et al. Family, Alzheimer's disease and negative symptoms. Int J Geriatr Psychiatry $2001 ; 16: 192-202$

11 Resnick B, Zimmerman SI, Magaziner J, et al. Use of the apathy evaluation scale as a measure of motivation in elderly people. Rehabil Nurs 1998;23:141-7.

12 Fogel B. The significance of frontal system disorders for medical practice and health policy. J Neuropsychiatry Clin Neurosci 1994;6:358-70.

13 Krupp BH. Ethical considerations in apathy syndromes. Psychiatr Ann 1997;27:50-4

14 Lieberman A, Ranhosky A, Korts D. Clinical evaluation of pramipexole in advanced Parkinson's disease: results of a double blind, placebo controlled, parallel-group study. Neurology 1997;49:162-8.

15 Guttman M, Jaskolka J. The use of pramipexole in Parkinson's disease: are its actions D3 mediated? Parkinsonism Rel Disord 2001;7:231-4.

16 Cummings JL. Cholinesterase inhibitors: a new class of psychotrophic compounds. Am J Psychiatry 2000;157:4-15.

17 Hutchinson M, Fazzini E. Cholinesterase inhibition in Parkinson's disease. J Neurol Neurosurg Psychiatry 1996;61:324-5.

18 Singer E. Social costs of Parkinson's disease. J Chron Dis 1973;26:243-54.

19 Litvan I, Mega MS, Cummings JL, et al. Neuropsychiatric aspects of progressive supranuclear palsy. Neurology 1996;47:1 184-9.

20 Siemers ER, Shekhar A, Quaid K, et al. Anxiety and motor performance in Parkinson's disease. Move Disord 1993;8:501-6.

21 Wenzel A, Holt CS. Dot probe performance in two specific phobias. $\mathrm{Br} J$ Clin Psychol 1999;38:407-10.

22 Cummings JL, Mega M, Gray K, et al. The Neuropsychiatric Inventory: comprehensive assessment of psychopathology in dementia. Neurology 1994;44:2308-14

23 Snaith P. Anhedonia: a neglected symptom of psychopathology. Psychol Med 1993;23:957-66

24 Meredith GE, Totterdell S. Microcircuits in nucleus accumbens shell and core involved in cognition and reward. Psychobiology 1999;27:165-86.

25 Braak H, Braak E, Yilmazer D, et al. Nigral and extranigral pathology in Parkinson's disease. J Neural Transm Suppl 1995;46:15-31.

26 Joyce JN, Ryoo H, Gurevich EV, et al. Ventral striatal D3 receptors and Parkinson's disease. Parkinsonism Rel Disord 2001;7:225-30.

27 Fibiger HC. The neurobiological substrates of depression in Parkinson's disease. J Can Sci Neurol 1984;11:105-7.

28 Goerent IK, Lawrence AD, Brooks DJ. Reward processing in the parkinsonian brain: an activation study using PET. Parkinsonism Rel Disord 1999;5:58.

29 Poewe W, Karamat E, Kemmler GW, et al. The premorbid personality of patients with Parkinson's disease: a comparative study with healthy controls and patients with essential tremor. In: Streifler MB, Korezyn AD, 
Melamed E, et al, eds. Advances in neurology. Parkinson's disease: anatomy, pathology, and therapy. New York: Raven Press 1990: 339-42.

30 Poewe W, Gerstenbrand F, Ransmayr G, et al. Premorbid personality of Parkinson patients. J Neural Transm 1983;19:215-24.

31 Menza MA, Mark MH, Burn DJ, et al. Personality correlates of [18F]dopa striatal uptake: results of positron-emission tomography in Parkinson's disease. J Neuropsychiatry Clin Neurosci 1995;7:176-9

32 Cloninger CR, Svrakic DM, Przybeck TR. A psychobiological model of temperament and character. Arch Gen Psychiatry 1993:50:975-90.

33 Brown RG, Marsden CD. Cognitive function in Parkinson's disease: from description to theory. Trends Neurosci 1990;13:21-9.

34 Kuzis G, Sabe L, Tiberti C, et al. Neuropsychological correlates of apathy and depression in patients with dementia. Neurology 1999;52:1403-7.

35 Castellon SA, Hinkin CH, Wood S, et al. Apathy, depression, and cognitive performance in HIV-1 infection. J Neuropsychiatry Clin Neurosci 1998;10:320-9.

36 Hoehn MM, Yahr MD. Parkinsonism: onset, progression, and mortality. Neurology 1967; 17:427-42.

37 Gotham AM, Brown RG, Marsden CD. Depression in Parkinson's disease: a quantitative and qualitative analysis. J Neurol Neurosurg Psychiatry 1986;49:381-9

38 Schwab RS, England AC. Projection technique for evaluating surgery in Parkinson's disease. In: Gillingham FJ, Donaldson MC, eds. Third symposium on Parkinson's disease. Edinburgh: Livingstone, 1969.

39 Marin RS, Biedrzycki RC, Firinciogullari S. Reliability and validity of the apathy evaluation scale. Psychiatr Res 1991;38:143-62.

40 Roth M, Huppert FA, Tym E, et al. CAMDEX: The Cambridge examination for mental disorders of the elderly. Cambridge: Cambridge University Press, 1988.

41 Folstein SE, Folstein MF. "Mini-mental state": A practical method for grading the cognitive state of patients for the clinician. J Psychiatr Res 1975; 12:189-98.

42 Hobson P, Meara J. The detection of dementia and cognitive impairment in a community population of elderly people with Parkinson's disease by use of the CAMCOG neuropsychological test. Age Ageing 1999;28:39-43

43 Nelson HE. A modified card sorting test sensitive to frontal lobe defects. Cortex 1976:12:313-24

44 Stroop JR. Studies of interference in spatial and verbal reactions. J Exp Psychol 1935;18:643-62.
45 Benton AL. Differential behavioural effects in frontal lobe disease. Neuropsychologia 1986;6:63-80.

46 Zigmond AS, Snaith RP. The hospital anxiety and depression scale. Acta Psychiatr Scand 1983:67:361-70.

47 Beck AT, Ward $\mathrm{CH}$, Mendelson $\mathrm{M}$, et al. An inventory for measuring depression. Arch Gen Psychiatry 1961;4:561-71.

48 Leentiens AF, Verhey FR, Luijckx GJ, et al. The validity of the Beck depression inventory as a screening and diagnostic instrument for depression in patients with Parkinson's disease. Move Disord 2000;15:1221-4.

49 Brown RG, MacCarthy B, Gotham AM, et al. Depression and disability in Parkinson's disease: a follow-up of 132 cases. Psychol Med 1988:18:49-55.

50 Snaith RP, Hamilton M, Morley S, et al. A scale for the assessment of hedonic tone the Snaith-Hamilton pleasure scale. Br J Psychiatry $1995 ; 167: 99-103$

51 Rabkin JG, Ferrando SJ, van Gorp W, et al. Relationships among apathy, depression, and cognitive impairment in HIV/AIDS. $J$ Neuropsychiatry Clin Neurosci 2000;1 2:451-7.

52 Starkstein SE, Petraca G, Chemerinski E, et al. Depression in classic versus akinetic-rigid Parkinson's disease. Move Disord 1998;13:29-33.

53 Cole SA, Woddard JL, Juncos JL, et al. Depression and disability in Parkinson's disease. J Neuropsychiatry Clin Neurosci 1996;8:20-5.

54 Cloninger CR. A systematic method for clinical description and classification of personality variants. Arch Gen Psychiatry 1987:44:573-88.

55 Menza MA, Golbe LI, Cody RA, et al. Dopamine-related personality traits in Parkinson's disease. Neurology 1993;43:505-8.

56 Frith CD. The cognitive neuropsychology of schizophrenia. Hove: Lawrence Erlbaum Associates, 1992.

57 Rodnitzky RL. Visual dysfunction in Parkinson's disease. Clin Neurosci 1998;5:102-6.

58 Owen AM, Evans AC, Petrides M. Evidence for a two-stage model of spatial working memory processing within the lateral frontal cortex: a positron emission tomography study. Cerebral Cortex 1996:6:31-8.

59 Le Bras C, Pillon B, Damier P, et al. At which steps of spatial working memory processing do striatofrontal circuits intervene in humans? Neuropsychologia 1999;37:83-90.

60 Cummings JL, Darkins A, Mendez M, et al. Alzheimer's disease and Parkinson's disease: comparison of speech and language disorders. Neurology 1988;38:680-4.

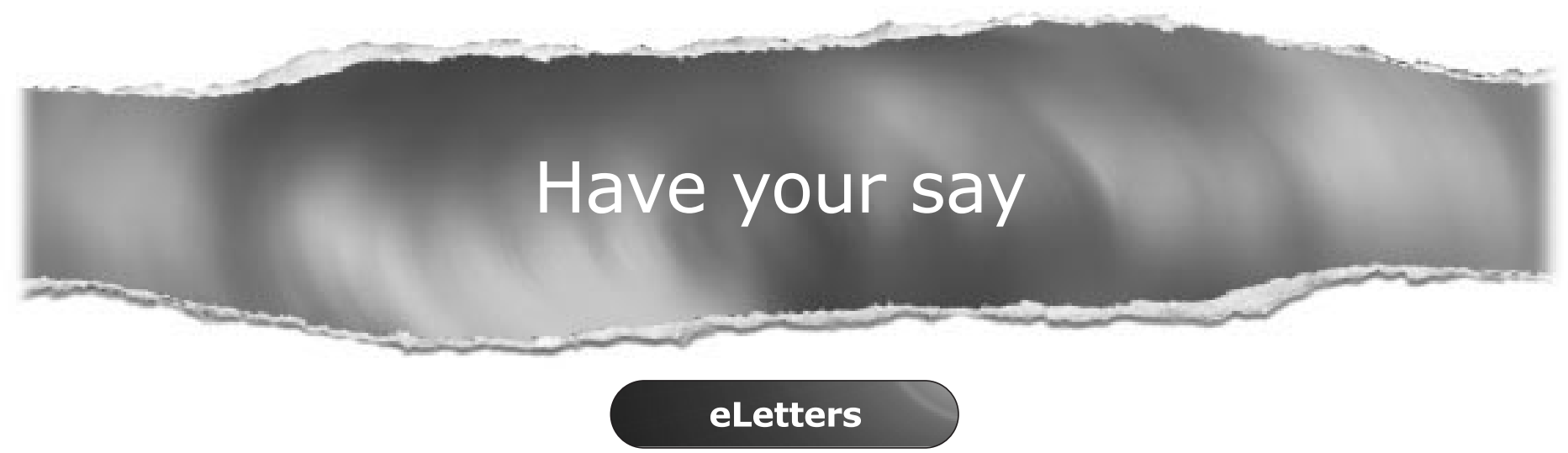

If you wish to comment on any article published in the Journal of Neurology, Neurosurgery, and Psychiatry you can send an eLetter using the eLetters link at the beginning of each article. Your response will be posted on Journal of Neurology, Neurosurgery, and Psychiatry online within a few days of receipt (subject to editorial screening).

\section{wWw.jnnp.com}

